\title{
Reconhecimento geoacústico da margem equatorial brasileira: área delta do rio Paranaíba (PI-MA)
}

\author{
Sarah Alves do Santos*1, Helenice Vital ${ }^{1}$, Andre Giskard Aquino da Silva1 ${ }^{1}$, Yoe Alain Reyes Pérez¹, ${ }^{1}$ GGEMMA/PPGG/UFRN
}

“Copyright 2021, SBGf - Sociedade Brasileira de Geofísica.

Este artigo foi preparado para apresentação no $17^{\circ}$ Congresso Internacional da Sociedade Brasileira de Geofísica, realizado no Rio de Janeiro, Brasil, de 16 a 19 de agosto de 2021.

O conteúdo deste artigo foi revisado pelo Técnico Comissão do $17^{\circ}$ Congresso Internacional da Sociedade Brasileira de Geofísica. As ideias e conceitos do texto são de recion de réch da SBGl, de seus dingentes ou membros. É proibida a reprodução eletrônica ou armazenamento de qualquer parte deste trabalho para fins comerciais sem consentimento por escrito da Sociedade Brasileira de Geofísica.

\begin{abstract}
This work was conducted in the brazilian equatorial margin: Parnaiba River Delta area (PI-MA), between the Tutóia and Luís Correia cities. The main objective was the hydroacoustic mapping of tidal channels of this area using side scan sonar and shallow seismic (chirp). 49 sonographic lines and 87 seismic lines were surveyed covering a total area of $24 \mathrm{~km}^{2}$, using small fisher boat. In the laboratory, the sonographic data processing included the use of filters in order to remove noises, that influenced directly in the low resolution and quality of the data. Shallow seismic processing was carried out following a flowchart formulated for the data under analysis, based on the information contained in the literature, and included the data, implementation of geometric corrections, spectral analysis, application of gains and filters, deconvolution and interpretation of seismic data. The results obtained from the processing and integration of acoustic data allowed the identification and sizing of different features present in the seabed, such as underwater dunes (width ranging from 7 to $15 \mathrm{~m}$ wide and heights between 0.10 to $1.2 \mathrm{~m}$ ), ripple marks, rocky outcrops and gas curtains. Therefore, this study intends to contribute to a better understanding of the evolution for a natural delta in response to climate change and sea level rise.
\end{abstract}

\section{Introdução}

Quando tratamos do estudo dos oceanos a partir da utilização de métodos geofísicos, são utilizados aqueles que se baseiam na emissão de ondas acústicas. Exemplos de métodos que podem ser utilizados na aquisição geofísica marinha, são: sísmica de alta resolução, batimetria e sonografia. Estes operam seguindo o mesmo preceito: a emissão, a transmissão e a reflexão de ondas geradas de forma natural ou artificialmente que se propagam nas diferentes camadas do fundo oceânico, nas quais apresentam propriedades elásticas distintas.

O método da sonografia utilizando o equipamento Side scan sonar, fornece informações sobre as características morfológicas e sedimentológicas do fundo do oceano. Através desse método podem ser vistas feições expressivas do relevo submerso, além da variação na distribuição superficial dos sedimentos. Após o processamento dos dados sonográficos, ou até mesmo durante o levantamento, quando o dado não apresenta ruídos, é possível a visualização de qualquer objeto que se encontra sobre o fundo do mar, tais como navios naufragados e estruturas de produção offshore (AYRES NETO, 2001, p.2).

O método sísmico, por outro lado, fornece dados sobre a disposição estrutural das camadas sedimentares abaixo do fundo marinho. Através de um registro sísmico é possível avaliar parâmetros como espessura de camadas, mergulho, presença de falhamentos, ocorrências de acumulações rasas de gás biogênico e deslizamentos submarinos (AYRES NETO, 2001, p.2). São utilizadas várias fontes para gerar uma onda acústica e, dependendo da fonte escolhida, podem-se ter informações mais detalhadas de subsuperfície.

Neste trabalho destaca-se a utilização da sonografia e da sísmica de alta resolução na caracterização das estruturas sedimentares abaixo do fundo marinho, em uma porção da margem equatorial brasileira, mas especificamente em canais da porção oeste do Delta do rio Parnaíba (PI-MA).

O rio Parnaíba está localizado entre os estados do Maranhão (MA), Piauí (PI) e Ceará (CE) entre as coordenadas $02^{\circ} 21^{\prime} \mathrm{S}$ e $11^{\circ} 06^{\prime} \mathrm{S}$ de latitude e $47^{\circ} 21^{\prime} \mathrm{W}$ e $39^{\circ} 44^{\prime} \mathrm{W}$ de longitude. A bacia de drenagem do rio em questão ocupa uma área total de $331.441 \mathrm{Km}^{2}$, sendo 249.497 Km² no Piauí, $65.492 \mathrm{Km}^{2}$ no Maranhão, 13.690 $\mathrm{Km}^{2}$ no Ceará e $2.762 \mathrm{Km}^{2}$ de área em litígio entre Piauí e Ceará. O rio Parnaíba é classificado como um rio do tipo perene, onde há sempre água fluindo em seu leito.

A Região do rio Parnaíba encontra-se fixada em duas grandes unidades estruturais: o escudo cristalino (15\% da área) e a Bacia sedimentar do Parnaíba (85\% da área). Caracteriza-se, principalmente, por sedimentos de cobertura Cenozóico (areias e argilas), rochas sedimentares do Mesozóico e Paleozóico (arenitos e argilitos) e Rochas Pré-Cambrianas (gnaises, granitos e migmatitos).

A área em que os levantamentos sísmicos e sonográficos foram realizados está localizada entre os municípios de Tutóia e Luiz Correía (Figura 1).

O levantamento sonográfico com o side scan sonar, totaliza uma área de $24 \mathrm{~km}^{2}$, resultante de 49 linhas adquiridas entre 31 de janeiro e 06 de fevereiro de 2009. No levantamento sísmico de alta resolução, utilizando o perfilador de subfundo Chirp, foram adquiridas o total de 87 linhas sísmicas (entre os dias 06 e 14 de outubro de 
2017), totalizando $249,31 \mathrm{~km}$. Todos os registros sísmicos foram processados, entretanto, apenas duas linhas foram escolhidas para serem interpretadas (Figura 1).

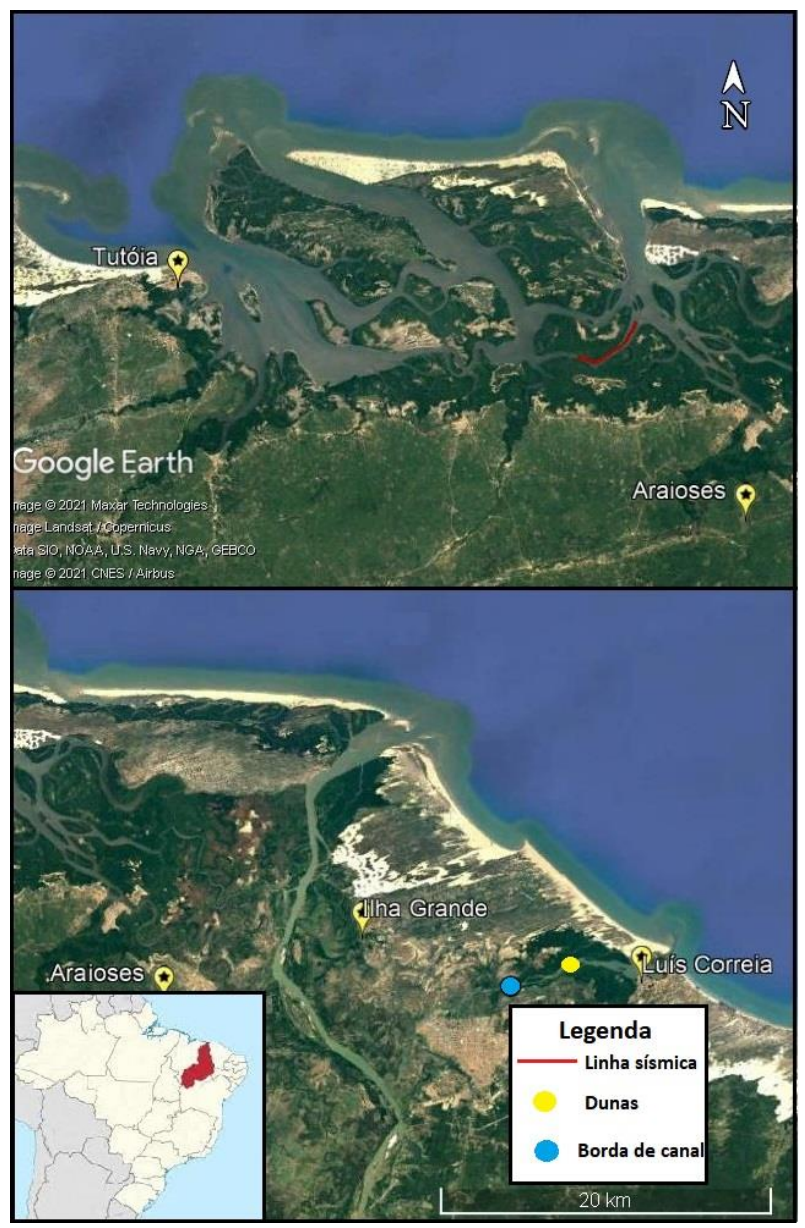

Figura 1 - Localização da área de estudo entre os municípios de Tutóia e Luís Correia no rio Parnaíba.

\section{Metodologia}

Ambos os levantamentos contém etapas de processamento semelhantes: leitura e a geometria do dado. A leitura do dado segundo escreveu Moreira (2017) é a etapa onde foi realizada a reformatação do dado para o formato interno do programa (SonarWiz e REFLEX). O dado foi importado para o software em questão e convertido do seu original formato no qual foi adquirido, para o formato interno do software. Já na geometria do dado foram inseridas as informações a respeito da localização de cada traço sísmico, através das coordenadas $\mathrm{X}$ e $\mathrm{Y}$ do dado.

\section{Sonar de Varredura Lateral}

Sonares de varredura lateral, geralmente, atuam em duas frequências de operação ditas de alta e baixa resolução, no qual dependem dos objetivos do levantamento e tipo do local a ser mapeado (MAYRINK, 2004). Altas frequências tais como $500 \mathrm{kHz}$ a $1 \mathrm{MHz}$, promovem excelente definição de imagem, entretanto, a área imageada é muito inferior quando comparadas com frequências mais baixas como $50 \mathrm{kHz}$ ou $100 \mathrm{kHz}$. Estas últimas, dão uma definição inferior, mas proporcionam áreas de imageamento muito maiores (VITAL, 2005).

Os dados foram processados no software SonarWiz v6 que permite a visualização da imagem bruta bem como seu processamento através da aplicação de diversos filtros, a fim de acentuar os diferentes aspectos da imagem (PEREIRA, 2016). A figura 2 mostra o fluxograma seguido para processamento dos sonogramas obtidos.

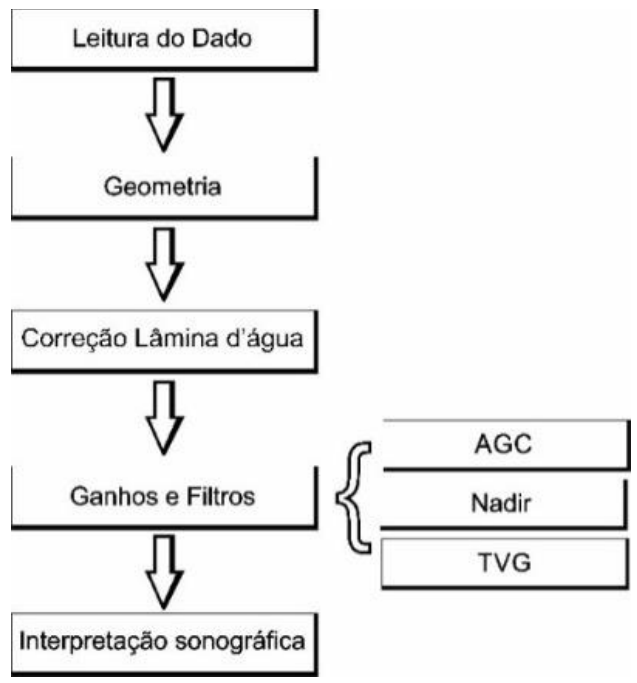

Figura 2 - Fluxo de processamento aplicado aos dados de sonar de varredura lateral.

\section{Correção da lâmina d'água}

A remoção da lâmina d'água foi realizada com o intuito de otimizar o tempo e a precisão do processamento, além de minimizar a saturação visual do dado (GOMES, 2009).

\section{Ganhos e Filtros}

Neste trabalho foram utilizados três tipos de filtros diferentes, foram eles: Time Varying Gain (TVG), Enable Nadir Filter (Nadir) e Automatic Gaisn Control (AGC). O TVG separa o dado em duas faixas paralelas, permitindo uma elevação maior em nível de contraste. Esse é um ganho típico baseado no tempo, que amplifica a energia dos sinais acústicos e fornece uma melhor qualidade aos dados, já que os pulsos acústicos emitidos se propagam para longe dos sensores, tornando-se cada vez mais espalhados, atenuados e absorvidos pelo meio.

Outro filtro utilizado foi o Enable Nadir filter o qual ajuda a suavizar o impacto visual da região do nadir (região central onde não há dados). Em alguns casos esse filtro pode não ajudar a suavizar a região central, pois nem todas as respostas de fundo marinho são iguais, cada uma tem sua particularidade.

O último ganho aplicado aos dados do side scan sonar, foi - Enable AGC que ajusta o nível do sinal (resolução e intensidade da imagem) conforme o sinal passa sobre 0 fundo.

\section{Sísmica Rasa}

Os dados sísmicos utilizados neste trabalho foram adquiridos com um perfilador de subfundo 3200-XS, fabricado pela EdgeTech (modelo SB0512i) e operou com frequências de $0.5-12 \mathrm{kHz}$. 
Os dados foram processados utilizando o software ReflexWin v6.0, no modo de processamento 2D- Data Analysis, seguindo a proposta adaptada da aplicação de fluxo de processamento (Figura 3) específico para dados sísmicos de alta resolução (GOMES; VITAL; MACEDO, 2010).

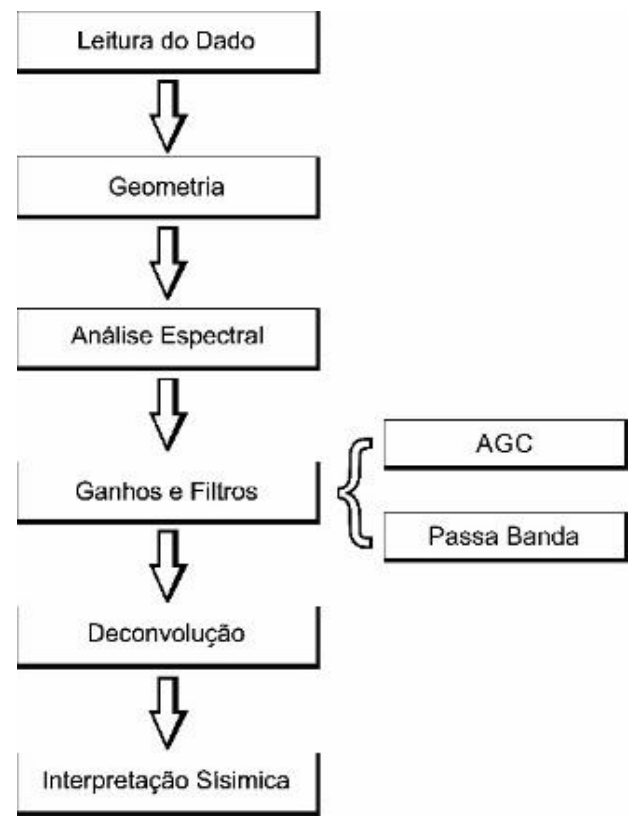

Figura 3 - Fluxo de processamento aplicado aos dados de sísmica de alta resolução.

\section{Análise Espectral}

A análise espectral foi utilizada para retirar as amplitudes anômalas dos dados sísmicos. Devido à emissão do sinal com uma dada frequência o sinal que retorna é um dado duplicado e este gera estruturas duplicadas e que não são reais.

\section{Ganhos e Filtros}

Com a propagação das ondas sonoras em subsuperfície ocorrem fenômenos associados à perda de amplitude dessa onda com o passar do tempo, o que ocasiona a perda do sinal e dificulta o reconhecimento dos refletores.

Esta etapa de aplicação de ganhos e filtros tem por finalidade corrigir a atenuação do sinal e eliminar alguns ruídos presentes no dado. Os filtros que operam em faixas de frequências são utilizados para remover fontes especificas de ruídos dado a frequência deste, sendo assim possível separar sinal de ruído (GOMES, 2009).

Foi utilizado o filtro de frequência passa banda no qual remove frequências indesejadas do dado eliminando ruídos e deixando passar apenas frequências dentro de uma banda especificada.

Foi aplicado ao dado o ganho AGC no qual faz uma distribuição equalizada das amplitudes (eixo y) dentro de uma janela de tempo já pré-definida. Esta distribuição é feita através de cálculos em torno da média das amplitudes que o dado apresenta.
Deconvolução

A deconvolução foi aplicada aos traços com o intuito de aumentar 0 espectro de frequência, atenuando as múltiplas e melhorando a resolução do dado para que não ocorram equívocos no momento da interpretação.

\section{Resultados}

Sonar de Varredura Lateral

As imagens foram analisadas em relação às tonalidades que apresentaram após a aplicação dos filtros, e podem ser correlacionáveis aos diferentes tipos de sedimentos e feições presentes no fundo marinho.

A figura 4A é referente ao dado bruto, enquanto a figura 4B ao dado processado, onde é possível observar algumas marcas que foram interpretadas como ripple marks, ou marcas de ondas simétricas, as quais são formadas pela oscilação do movimento de ondas em ambiente subaquático. $\mathrm{Na}$ figura $4 \mathrm{~B}$ tais marcas estão circuladas pela linha em vermelho.

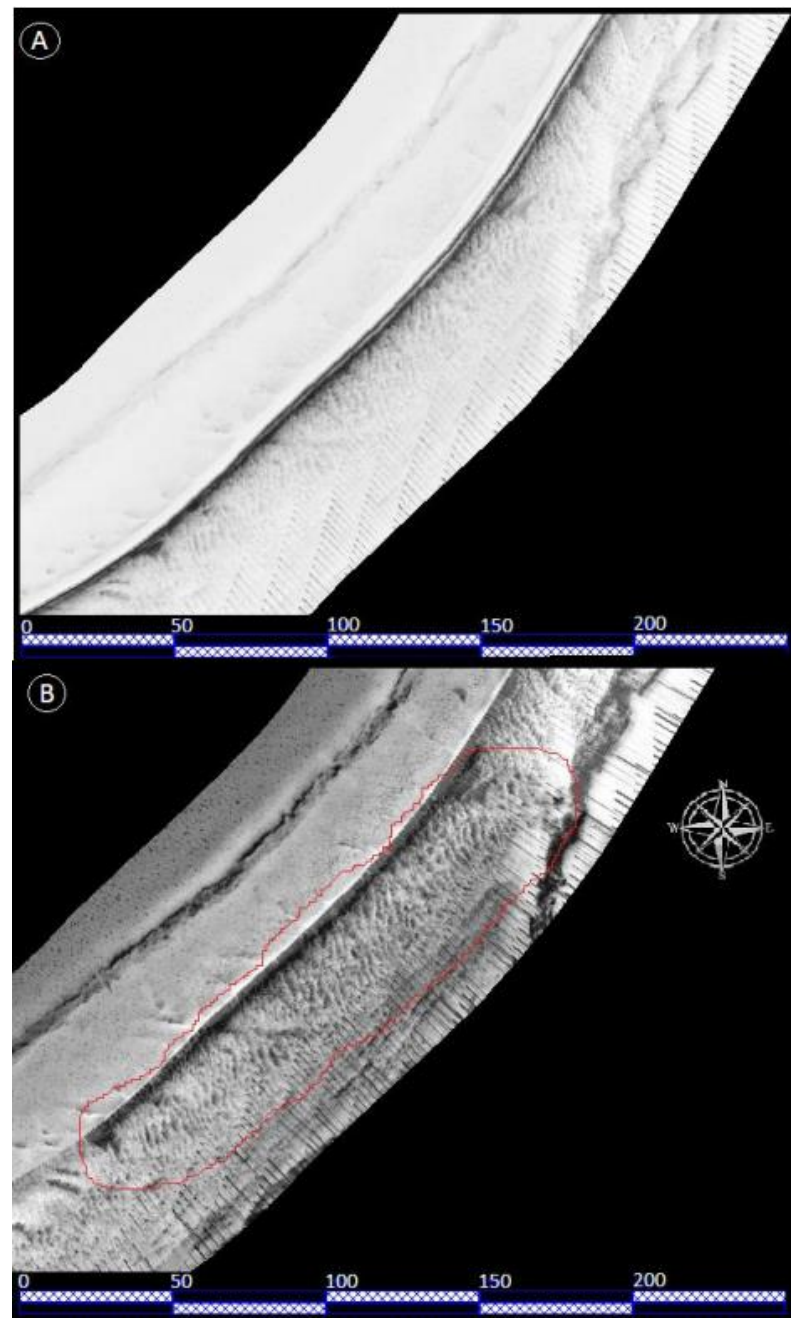

Figura 4 - Indicação de feições características de ripple marks. A) dado bruto, B) dado processado 
Após aplicação dos filtros é possível visualizar em cima da borda de canal (Figura 5, lado B), estruturas similares a dunas do tipo acanaladas que indicam a direção do fluxo da água do local, no caso, a água está fluindo na direção Nordeste (NE) e desembocando no mar. As dunas aqui presentes têm larguras que variam entre 7 e 15 metros e alturas variantes entre 0,10 a 1,2 metros.

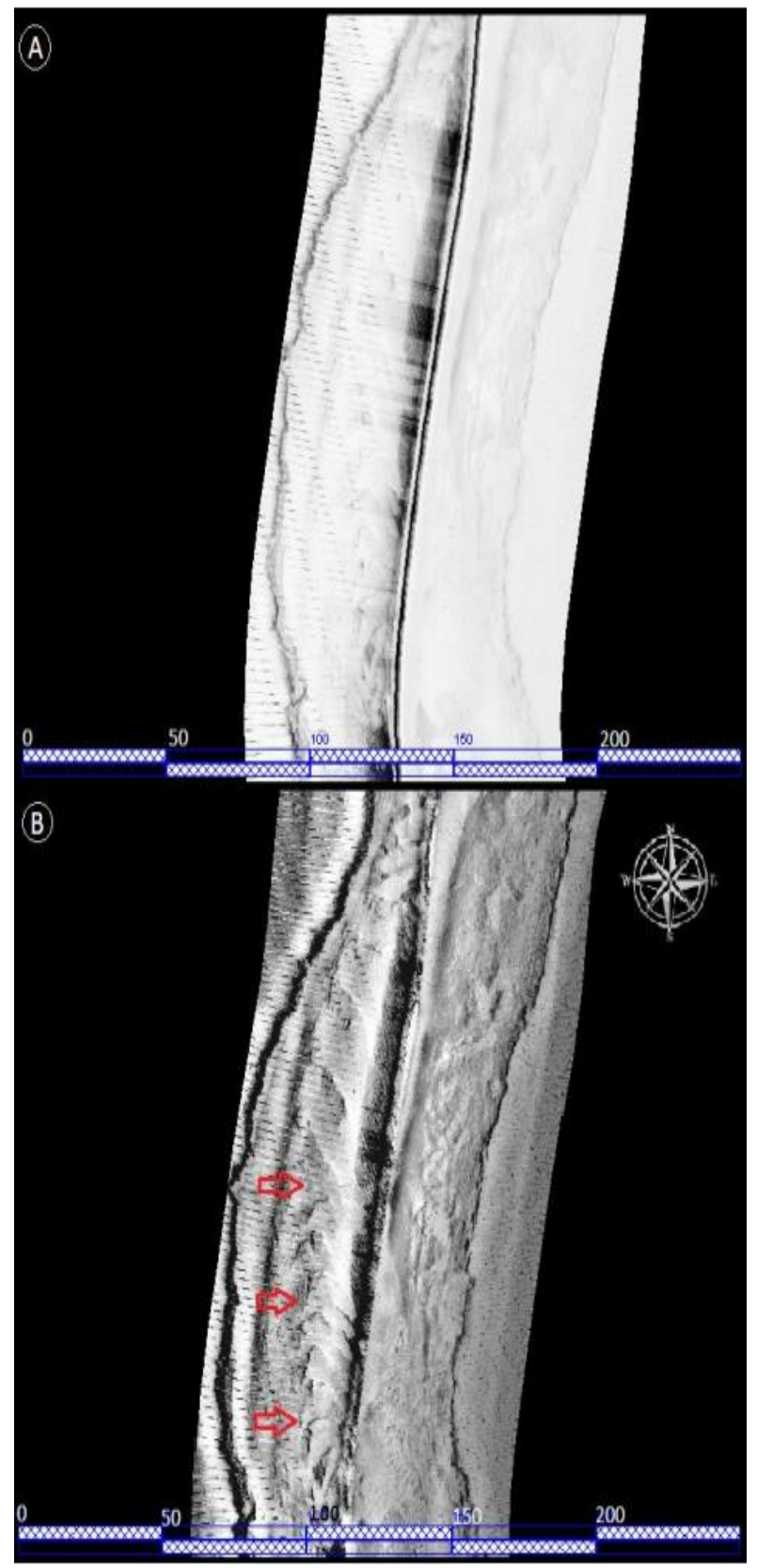

Figura 5 - Lado B da figura mostrando marcas que são características de dunas do tipo acanaladas.

A figura 6 mostra a presença de feições associadas a bordas de canais presentes na área, com largura de 64 metros indicadas por setas vermelhas na figura 6 . Tais marcas são indicativas dos mangues que a região apresenta.

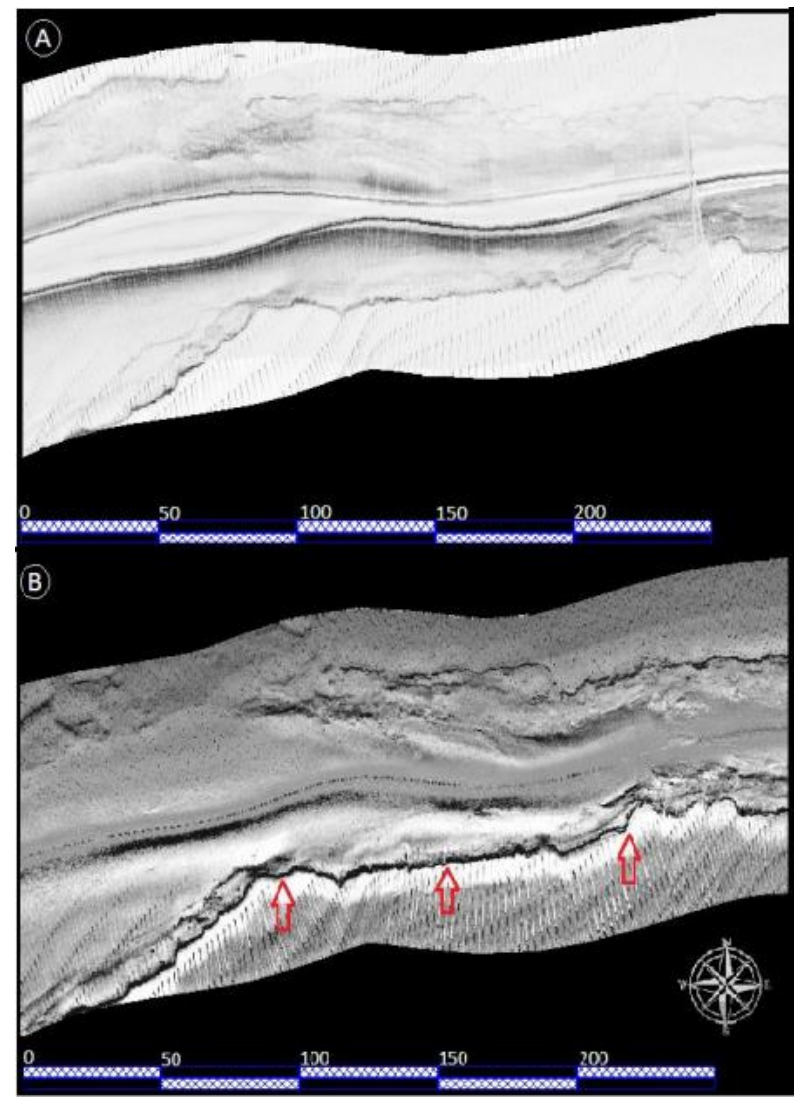

Figura 6 - Lado B da figura mostrando marcas que são características de bordas de canal.

\section{Sísmica Rasa}

As interpretações foram feitas utilizando o software Petrel da Schlumberger que permitiu a importação e visualização das imagens no formato SEGy e permitiu a aplicação de atributos sísmicos sendo o mais importante o TecVA (Bulhões e Amorim, 2005) para melhorar o aspecto serrilhado da imagem e destacar as feições presentes nas seções sísmicas.

A figura 7, cuja localização é mostrada no mapa da figura 1, apresenta no lado $B$ da linha um trecho da seção sísmica interpretada, onde podemos identificar a presença de camadas possivelmente referentes ao período Quaternário, da era cenozoica. É possível visualizar a formação de paleocanais (canais um dia ativos, porém no atual presente estão inativos).

Podemos identificar na figura 7 os chamados de barras de meandros ou de pontal, segundo escreveu Jordan e Grotzinger (2013, p.507) elas ocorrem da seguinte forma: a erosão ocorrerá nas partes côncovas das curvas de meandro (lado erosivo), onde a velocidade da corrente é mais alta. A deposição de sedimentos, por outro lado, ocorrerá ao longo das partes convexas do meandro interno (lado deposicional), onde a velocidade é baixa. Tal deposição de sedimentos resulta em barras expostas, chamadas de barra de pontal. Como as correntes sinuosas estão continuamente erodindo o lado erosivo do meandro, as curvas e o deposito de sedimentos ao longo do lado deposicional do meandro tendem a migrar lateralmente na 
planície de inundação, formando assim a estrutura de paleocanais vistos na figura 7 .

Ainda foi possível visualizar a presença de uma camada (b) que possivelmente possa ser um paleocanal presente na área, em $\mathrm{C}$ vemos a presença da lâmina d'água e nela o registro de plumas acústicas, que possivelmente são referentes a concentração de gás biogênico que ocorre na área.

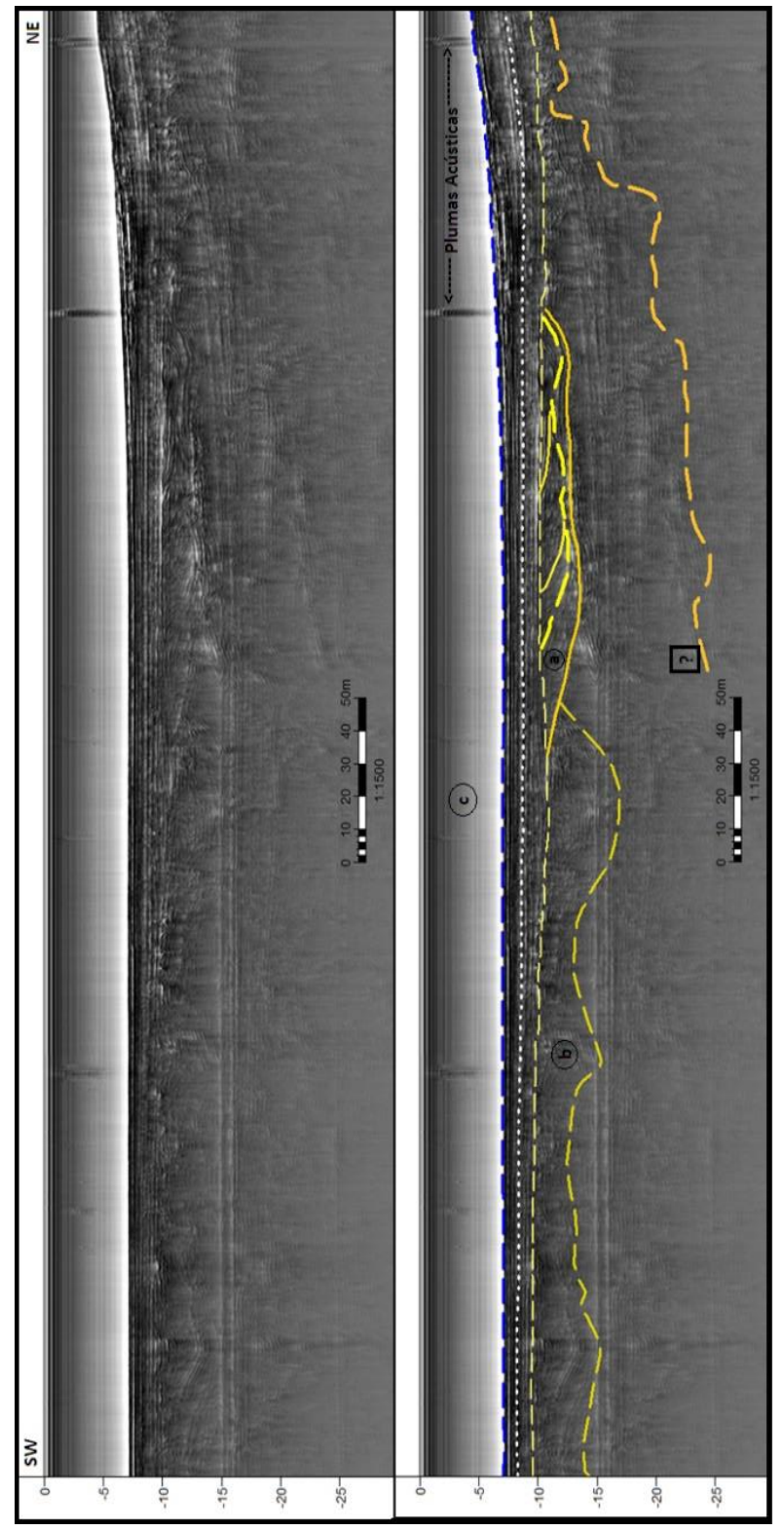

Figura 7 - Registro sísmico de alta resolução. A) dado bruto, B) dado processado, mostrando a ocorrência de paleocanais $(a, b)$, e a ocorrência de plumas acústicas na lâmina d'água.

A figura 8 mostra a presença de dois paleocanais; o paleocanal 1 sendo o mais antigo e o 2 o mais novo. Acima dos dois paleocanais observa-se uma camada de sedimentos com estratificação plano paralela recobrindoos, indicando que o local apresenta fluxo laminar, ambiente propicio a deposição de sedimentos finos (argila ou lama).

A figura 8 também indica duas perturbações no registro sísmico, em forma de colunas (ou cortinas), uma com 44,4 metros de comprimento e a outra com 12,5 metros de comprimento. Estas perturbações colunares são similares às descritas por Taylor DI (1992) e Garcia Gil S (2002). O que nos levou a considerar que as perturbações colunares, na verdade, são colunas acústicas. Ainda segundo Taylor DI (1992) e Garcia Gil S (2002) as colunas acústicas apresentam comprimento de até $50 \mathrm{~m}$ e indicam uma alta taxa de sedimentação.

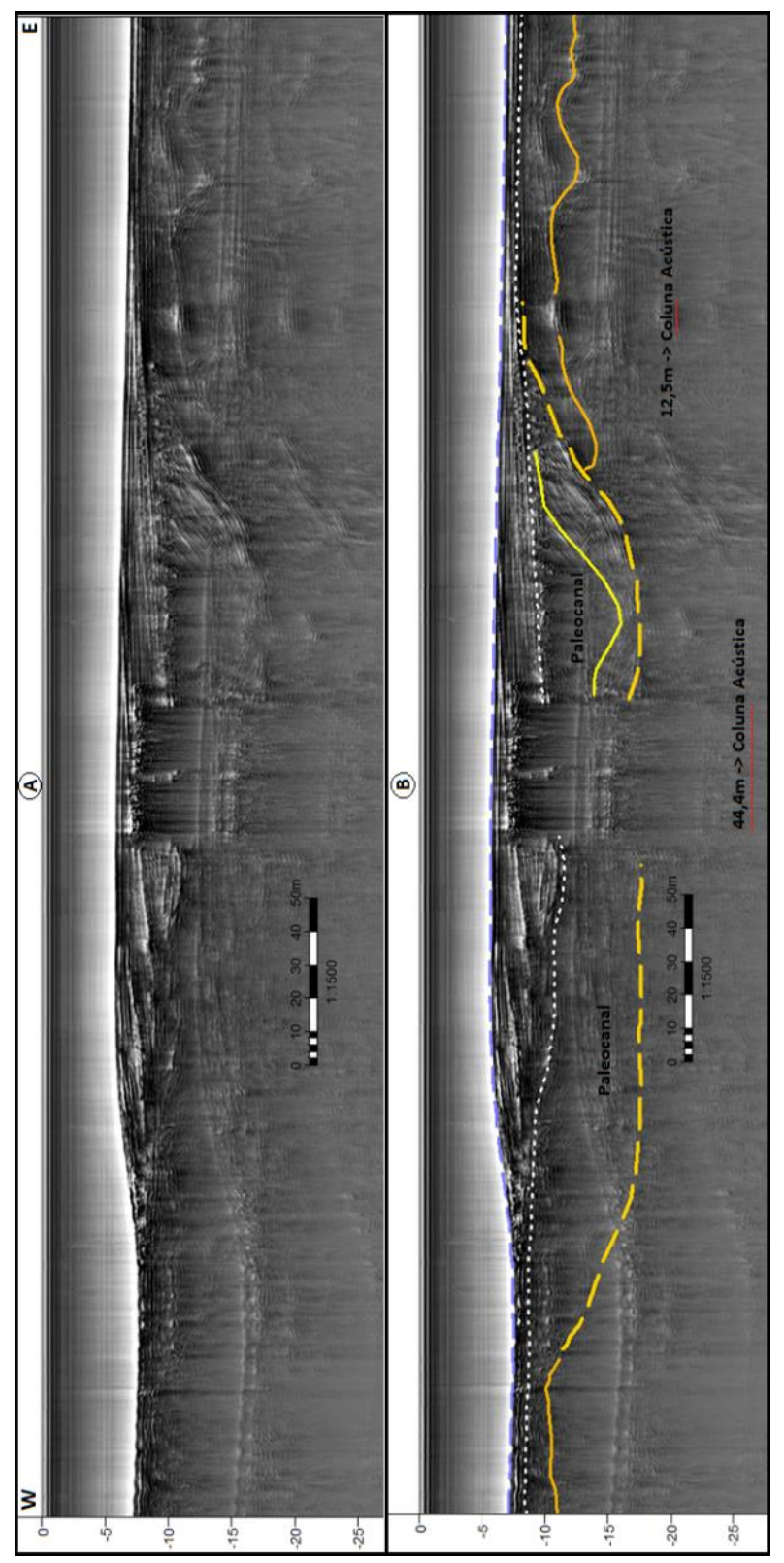

Figura 8: Seção sísmica indicando a presença de dois paleocanais e duas colunas acústicas, uma com 12,5 metros e a outra com 44,4 metros de comprimento. A) dado processado e B) dado processado e interpretado. 


\section{CONCLUSÕES}

O levantamento sonográfico e o levantamento sísmico permitiram, pela primeira vez, a visualização das feições de fundo presentes nos canais da região do delta do Rio Parnaíba (PI/MA). Foram identificados com o uso do Sonar de varredura lateral diferentes feições de fundo, incluindo dunas com larguras variando entre 7 a $15 \mathrm{~m}$ e alturas entre 0,10 a 1,2 m, bordas de canais com larguras de até $64 \mathrm{~m}$ e marcas de ondas. O processamento dos dados foi de fundamental importância para identificação com clareza das feições presentes. O fluxo de processamento escolhido foi eficiente no aumento da razão sinal/ruído. O processamento da sísmica rasa se provou eficiente no aprimoramento das imagens brutas, tornando assim possível identificar colunas acústicas. Além disso, foram identificados vários paleocanais, os quais indicam uma intensa modificação na direção e hidrodinâmica local. A integração dos dados de sonar de varredura lateral com os dados de sísmica rasa permitiram a visualização, identificação e dimensionamento das feições geomorfológicas e do substrato marinho pertencente ao delta do rio Parnaíba. Estes resultados contribuem para uma melhor compreensão da evolução geológica de um delta natural, em resposta às mudanças climáticas e à elevação do nível do mar.

\section{Agradecimentos}

À Universidade Federal do Rio Grande do Norte pela infraestrutura necessária ao desenvolvimento deste trabalho de conclusão de curso - TCC da primeira autora, sob orientação e co-orientação dos $2^{\circ}$ e $3^{\circ}$ autores; ao apoio financeiro do Conselho Nacional de Desenvolvimento Científico e Tecnológico - CNPq e da Coordenação de Aperfeiçoamento do Ensino Superior CAPES, através do Instituto Nacional de Ciência e Tecnologia "Ambientes Marinhos Tropicais"- INCT AmbTropic (CNPq - FAPESB - CAPES) e dos projetos: (88881.068034/2014-01, CAPES CSF-PVE 2014 3 a chamada), Auxilio PQ Processo no311413/2016-1 e Programa PIBIC (UFRN-CNPq).

\section{Referências}

AYRES NETO, Arthur. Uso da sísmica de reflexão de alta resolução e da sonografia na exploração mineral submarina. Brazilian Journal Of Geophysics. Niterói, Rj, p. 1-16. maio 2001.
BULHÕES, E. M; AMORIM, W. N. Princípio da Sismocamada Elementar e sua aplicação à Técnica de Volume de Amplitudes (tecVA). In: Ninth International Congress of the Brazilian Geophysical Society, 6 p, 2005.

GARCIA-GIL S, VILAS F \& GARCIA-GARCIA A. 2002. Shallow gas features in incised-valley fills (Ría de Vigo, NW Spain): a case study. Continental Shelf Researche, 22: $2303-2315$

GOMES, Moab Praxedes. AQUISIÇÃO, PROCESSAMENTO E ANÁLISE DE DADOS DE SÍSMICA DE ALTA RESOLUÇÃO NA PLATAFORMA CONTINENTAL NORTE DO RIO GRANDE DO NORTE: VALE INCISO DO RIO AÇU. 2009. 72 v. Dissertação (Mestrado) - Curso de Programa de Pós-graduação em Geodinâmica e Geofísica, Ciência Exatas e da Terra, Universidade Federal do Rio Grande do Norte, Natal, 2009. Cap. 5.

GOMES, Moab Praxedes; VITAL, Helenice; MACEDO, José Wilson de Paiva. FLUXO DE PROCESSAMENTO APLICADO A DADOS DE SÍSMICA DE ALTA RESOLUÇÃO EM AMBIENTE DE PLATAFORMA CONTINENTAL. Exemplo: Macau-RN. Revista Brasileira de Geofísica, v. 29(1): p.173-186, dez. 2010.

JORDAN, Tom; GROTZINGER, John. PARA ENTENDER A TERRA. 6. ed. Porto Alegre: Bookman, 2013. 738 p.

MOREIRA, Myrli Andrade. MAPEAMENTO DE FEIÇÕES DEPOSICIONAIS E EROSIVAS NO ESTUÁRIO DO RIO AÇU/RN (NE DO BRASIL) UTILIZANDO O MÉTODO HIDROACÚSTICO DE ALTA RESOLUÇÃO E SONDAGEM GEOLÓGICA. 2016. 90 f. Dissertação (Mestrado) - Curso de Programa de Pós-graduação em Geodinâmica e Geofísica, Ciência Exatas e da Terra, Universidade Federal do Rio Grande do Norte, Natal, 2017. Cap. 2.

PEREIRA,T.R.B.Aplicação De Métodos Hidroacústicos NaClassificação Textural Do Fundo Marinho. Dissertação (Mestrado em Geodinâmica e Geofísica) - Programa de Pós-Graduação em Geodinâmica e Geofísica, Universidade Federal do Rio Grande do Norte, 66p, 2016.

TAYLOR DI. 1992. Nearshore shallow gas around the UK coast. Continental Shelf Research, 12: 1135-1144.

VITAL, Helenice et al. Oceanografia geológica e geofísica da plataforma continental brasileira. In: SOUZA, Celia Regina de Gouveia et al. Quaternário do Brasil. São Paulo: Holos, 2005. p. 153-173. 